\title{
Retinoblastoma genetics: A Survey
}

\author{
V.Sah \\ SDIDTS, India
}

\begin{abstract}
The prototypical hereditary cancer is retinoblastoma. With an approximate 1500 new cases each year, India has the highest prevalence of retinoblastoma in the world. Recent findings in retinoblastoma genetics are discussed, with an emphasis on knowledge that is clinically relevant to patients. There is also a discussion of the Indian literature on retinoblastoma clinical genetics, as well as issues and prospective paths. The study ends with suggestions to assist physicians in incorporating and applying retinoblastoma genetics into their practice
\end{abstract}

\section{INTRODUCTION}

The research of retinoblastoma has added significantly to our knowledge of cancer heritability since it was the first tumor to be reported to have genetic origins $[1,2,3,4,5]$. Although most of the genetic science focuses on elucidating the disease's specific molecular growth, there are significant findings that can be translated directly to health treatment and help people live longer lives. India is home to about $20 \%$ of the world's retinoblastoma patients. This has significant consequences for India's healthcare sector, not just in terms of the cost it imposes, but also in terms of the prospects for health and science that arise. The key goals of this analysis article are twofold. It begins by reviewing recent developments in retinoblastoma genetics, with a particular emphasis on knowledge that is important and pertinent to patient care. Second, it provides a scoping analysis to determine the scope and depth of clinical genetic study on retinoblastoma in India. The study ends with guidelines to assist healthcare professionals in implementing and translating retinoblastoma genetics in their clinics so that Indian families afflicted by the disease will learn from the most up-to-date relevant research.

\section{METHODS}

A scan of PubMed (accessed January 15, 2014) was conducted to find appropriate and timely papers for the first part of this study. The terms "retinoblastoma biology," "RB1 mutation," "retinoblastoma genetic research," and "retinoblastoma genetic therapy" were used often. Hand-searching turned up further reviews on retinoblastoma and retinoblastoma genetics. Papers that did not specifically address retinoblastoma were not included. "Clinical" (primary research on patients and/or patient outcomes), "Basic" (primary research on biological causes of retinoblastoma), "Genetic" (primary research on retinoblastoma medical genetics/mutation detection), or "Review" (primary research on retinoblastoma medical genetics/mutation detection) or "Review" (primary research on retinoblastoma medical genetics/mutation (nonprimary research, review articles). In order to find additional clinical genetics papers that may have been missing in the PubMed search, a Google search with the keywords "retinoblastoma genetics in India" was conducted (January 21, 2014). Data from clinical genetics papers are used to measure the sensitivity of RB1 mutation testing for unilateral (UNI) and bilateral (BI) cases where available. The number of patients recorded on, their laterality, the number of blood specimens checked (BI), the number of M1 mutations detected (BI), the number of tumor specimens tested (UNI), and the number of M1/M2 pairs of mutations found were all included in this data (UNI).

\section{RECENT STUDY}

The first tumor of which the molecular nature of cancer was discovered was retinoblastoma. (1), (2), (3), (4), (5), (6), (7), Even though genetic mutations affect all retinoblastoma tumors, this does not indicate that all patients have contracted the disorder or that all specimens are passable to the next generation. Recent research has shown that retinoblastoma tumors may vary in their mutagenic pathways from regular to malignant cells; for example, some retinoblastoma tumors are induced by RB1 mutations [1,2,3,4,5], whereas others are caused by MYCN gene amplification. [number six] It's important to think about retinoblastoma genetics in terms of heritability (heritable or nonheritable) and laterality to grasp the disease's genetics (UNI or BI). 
Individuals with heritable retinoblastoma (48\%) have a germline mutation in the RB1 tumor suppressor gene, which makes them more likely to grow not only retinal tumors (UNI, 7\%), but also pineal tumors (trilateral) and second cancers later in life. The loss of the second RB1 allele in a sensitive retinal cell causes the majority of heritable retinoblastoma patients to grow retinal tumors, which may be benign (retinoma) or malignant (retinoblastoma). It is also common to have heritable retinoblastoma and not grow retinal tumors (1\%); however, these people are also at risk for cancer later in life. About $10 \%$ of people with heritable retinoblastoma have acquired the RB1 mutation from one of their parents. This ensures that the bulk of people with hereditary retinoblastoma are the first in their families to be diagnosed. About $4 \%$ of people with heritable retinoblastoma are mosaic for the RB1 mutation, which means the mutation happened during embryogenesis and only impacts a small portion of the germline. (8), (9) Mosaic people could not have acquired their mutation (otherwise, the RB1 mutation would be present in both of their cells), but their condition could be passed on to future generations. Mosaicism could theoretically reduce the risk of transfer to the next generation; moreover, there is currently no credible evidence to reliably quantify possible reduced risks. The majority of people with nonheritable retinoblastoma (52\%) have regular RB1 genes in their germline. Tumors are often UNI and unifocal, and they arise through one of two mechanisms: (1) loss of both copies of RB1 in a susceptible retinal cell $(51 \%)$ or (2) amplification of the MYCN oncogene in a susceptible retinal cell (1 percent ). Since the hereditary abnormalities are somatic, neither these people nor either of their families, current or potential, are at a greater risk of cancer later in life.

\section{PROGRESSION}

The lack of two RB1 alleles in a vulnerable retinal cell causes genomic instability, which contributes to copy number changes in many other genes in the majority of retinoblastoma tumors: MDM4, KIF14, MYCN, DEK, and E2F3 copy number increases, while CDH11 copy number decreases. [nine] The degree of genomic instability separates benign retinoma (less genomic instability) from malignant retinoblastoma (more genomic instability) (more genomic instability). [nine] Deregulation of microRNAs, aberrant methylations, single nucleotide polymorphisms, and variable gene expression are some of the other genetic changes that occur throughout the growth of retinoblastoma; these have all been thoroughly studied elsewhere. (12) Beyond the initial activation of the MYCN oncogene, nothing is understood regarding the production of MYCNamp tumors.[6] Is MYCN amplification the only genomic event that causes these tumors to become malignant? Are MYCNamp tumors different from RB1/retinoblastomas in terms of cell origin? These questions remain unanswered, and further research is required. Although research into the genetic development of retinoblastoma beyond the triggering mutational event may one day contribute to targeted therapies for retinoblastoma patients, relatively little of this study is currently applicable to clinical treatment. Instead, it is critical to determine if they have heritable retinoblastoma; additional details about the triggering case will then be used to guide treatment. For example, while all BI patients have heritable retinoblastoma, accurate risk estimation in family members and potential descendants is impossible without genetic tests to identify the RB1 mutation. This is especially significant for UNI patients, who may benefit from accurate genetic detection to distinguish between heritable and nonheritable events, as well as RB1/versus MYCNamp retinoblastoma.

Knowing the molecular genetic make-up of retinoblastoma tumors and an individual's mutation carrier status allows for better screening and care of the patient and their family, thus removing this possibility eliminates unwanted medical trips and anxiety. Two resources are needed for this to occur: comprehensive genetic tests performed by a capable lab and responsive and accurate counselling to convey the knowledge to the patient and relatives.

The method used to discover and analyze the genetic outcome is just as effective as the technique used before it. This begins with the processing of the sample (blood and/or tumor). This is especially significant for tumors, which can only be sampled once, unlike blood, after enucleation and until the remainder of the eye is sent to histopathology. A technique for optimizing tumor sampling for genetics while preserving the quality of the specimen for future histopathological examination has been proposed. (\#13) It's critical to choose the right storage media to ensure that you get the most out of your DNA and potentially RNA extraction. Retinoblastoma genetics laboratories may advise on the best methods for collecting, storing, and transporting both tumor and blood.

About any single retinoblastoma patient may have a different RB1 mutation (excluding of course, those who have MYCNamp retinoblastoma). 14 and 15 There are just a few RB1 mutations that are recurring. This suggests that, rather than a clear screen for identified mutations, genetic research in the proband is still a voyage of exploration. Broad and tiny deletions, point mutations, insertions, translocations, deep intronic splice mutations, and promoter methylation may all cause harm to the RB1 gene. 14 and 15 This necessitates the usage of a variety of methods in the quest for the offending mutant. $[15,16,17]$ If you're looking for a unique way to express yourself, However, after the actual mutation in the proband has been detected, descendants and potential children may be conveniently tested for the known mutation. It's worth noting that we realize the highest potential result with all formulas is 100 percent. That is, 100 percent of BI patients have a 
germline RB1 mutation in their blood, and 100 percent of UNI patients (except MYCNamp cases) have mutations in all tumor RB1 alleles. This isn't to suggest that laboratories should hope to achieve $100 \%$ sensitivity. That could be difficult to achieve using existing methods and technologies. The degree of divergence from 100 percent sensitivity, on the other hand, may be used to assess how effectively a laboratory detects predicted RB1 mutations. When reading a "no mutation detected" finding, test sensitivity is crucial. How can you tell if a "no mutation detected" finding is attributable to the lab's inability to identify an emerging mutation or whether the individual being checked isn't a mutation carrier? Consider a UNI recipient who has no family history with the disease. The specialist would proceed to monitor the child in case tumors form in the unaffected eye without genetic tests to validate or rule out the risk of heritable retinoblastoma. Family members of the infant may already be assumed to be in danger. Imagine the that child's tumor and blood are also checked. Two RB1 mutations are identified in the tumor and then tested for in the blood to see whether the infant has heritable (one of the two tumor RB1 mutations is observed in the blood) or nonheritable retinoblastoma (none of the two tumor RB1 mutations is detected in the blood).

Consider the scenario that the laboratory just receives a blood sample when the surgeon has decided to treat the infected eye (thus no tumor sample is available). A blood sample is examined, but no mutations are discovered. Is the kid a nonheritable case, or did the laboratory miss an RB1 mutation in the germline? "So long as the laboratory has shown that 90 percent of RB1 mutations may be identified," one advice from the Canadian Guidelines for Retinoblastoma Care says, "a negative outcome indicates chances are minimal enough that tests under anaesthesia can be avoided." [nineteen] Furthermore, the amount of tumors examined by a particular lab has an effect on understanding susceptibility. A high sensitivity with just a few specimens tested is unreliable, just as a poor sensitivity with several specimens tested indicates a reduced capacity to accurately diagnose RB1 mutations. The sensitivity of RB1 mutation research has been stated to be as high as 96\%. [nine]. MYCNamp retinoblastoma is a comparatively recent form of retinoblastoma. [number six] This form of retinoblastoma can be quickly detected using a simple copy number test of tumor DNA for the MYCN gene. Because of its rarity, it's understandable that this procedure will be used after genetic tests for RB1 failed to show a mutation in a UNI tumor, or whether histology showed the existence of neuroblastoma-like histology, which is a hallmark of MYCNamp retinoblastoma.

\section{CONCLUSION}

The key goal of this paper was to examine existing awareness of retinoblastoma genetics as it applies to patient treatment and compare it to reported proof of retinoblastoma genetic testing as it is used in India. Is genetic testing for retinoblastoma part of the quality of treatment in India? Although there are laboratories that offer retinoblastoma genetic testing, this analysis did not take into account any potential obstacles (social, economic, etc.) that could preclude a family from taking advantage of the program. It was often overlooked because certain families would be able to obtain retinoblastoma testing from overseas laboratories. Non-academic reports from commercial laboratories that could provide retinoblastoma genetic testing were not reviewed, and neither were institutional or technical constraints that could preclude a laboratory from obtaining reliable, high-quality results. This study of scholarly studies on retinoblastoma genetics in India does, however, point to the presence of a "know-do" gap: In India, latest retinoblastoma genetics information does not seem to be fully applied. Although a few Indian laboratories have reported their experiences with RB1 genetic research, the consistency of the results differs, making it difficult to determine how accurate various institutions' testing. Reliability of outcomes is critical for practicing physicians care for children with retinoblastoma, since it influences their medication and surveillance options.

\section{REFERENCES}

[1] Bowman, M., Debray, S. K., and Peterson, L. L. 1993. Reasoning about naming systems. Ding, W. and Marchionini, G. 1997 A Study on Video Browsing Strategies. Technical Report. University of Maryland at College Park.

[2] Fröhlich, B. and Plate, J. 2000. The cubic mouse: a new device for three-dimensional input. In Proceedings of the SIGCHI Conference on Human Factors in Computing Systems

Tavel, P. 2007 Modeling and Simulation Design. AK Peters Ltd.

[3] Stott JRR. Orientation and disorientation in aviation. Extreme Physiology \& Medicine. 2013; 2: 2.doi: 10.1186/2046-7648-2-2?optIn=true.

[4] Newman RL, Rupert AH. The magnitude of the spatial disorientation problem in transport airplanes. Aerosp Med Hum Perf. 2020; 91(2): 65-70.doi: 10.3357/AMHP.5442.2020.

[5] Gillingham KK. A primer of vestibular function, spatial disorientation, and motion sickness. Aeromedical Reviews. 1966; 4: 1-80.

[6] Gillingham KK. The spatial disorientation problem in the United States Air Force. Journal of vestibular research: equilibrium \& orientation. 1992; 2(4): 297-306. 
[7] Lawson B, McGrath B, Rupert A, Thompson LI, Brill JC, Kell AM. A countermeasure for loss of situation awareness: Transitioning from the laboratory to the aircraft. 2016 IEEE Aerospace Conference, Big Sky, USA, 2016

[8] Belcastro CM, Foster JV, Shah GH, Gregory IM, Cox DE, Crider DAet al. Aircraft loss of control problem analysis and research toward a holistic solution. Journal of Guidance, Control, and Dynamics. 2017; 40(4): 733-75.doi: 10.2514/1.G002815.

[9] Heinle TE, Ercoline WR. Spatial disorientation: Causes, consequences, and countermeasures for the USAF. The RTO HFM Symposium, AIR FORCE RESEARCH LAB WRIGHT-PATTERSON AFB OH HUMAN EFFECTIVENESS DIRECTORATE, La Coruña, Spain, 2003

[10] Dixon JB, Endsley T, Clark TK. A mathematical model-based metric of spatial disorientation for use in triggering active countermeasures. Proceedings of the Human Factors and Ergonomics Society Annual Meeting. 2019; 63(1): 1724-8.doi: 10.1177/1071181319631027.

[11] Holmes SR, Bunting A, Brown DL, Hiatt KL, Braithwaite MG, Harrigan MJ. Survey of spatial disorientation in military pilots and navigators. Aviat Space Environ Med. 2003; 74(9): 957-65.

[12] R. Patel, A. Kunche, N. Mishra, Z. Bhaiyat, R. Joshi, Comparative review of existing mobile payment systems, International Journal of Applied Engineering Research, 10 (2015), pp. 16873-16884

[13] Patel, R., Kunche, A., Mishra, N., Bhaiyat, Z., \& Joshi, P. R. (2015). Paytooth - a cashless mobile payment system based on Bluetooth. International Journal of Computer Applications, 120, 38-43. https://doi.org/10.5120/214124450 .

[14] Buxton, E. K., Vohra, S., Guo, Y., Fogleman, A., \& Patel, R. (2019). Pediatric population health analysis of southern and central Illinois region: A cross sectional retrospective study using association rule mining and multiple logistic regression. Computer methods and programs in biomedicine, 178, 145-153.21(2): 135-52.doi: 10.1080/10508414.2011.556458.

[15] Rushabh Patel and Rahul Raghvendra Joshi, Evolution \& Envisage of Mobile Network, International Journal of Scientific Engineering and Technology Research ISSN 2319-8885 Vol.03, Issue.01 (January-2014), pp. 0136-0143.

[16] Lucas J. Vespa et al, A Novel Reinforcement Sample Learning Strategy for Convolution Neural Network in Computer Aided Diagnosis System for Breast Cancer, SIIM 2017 Scientific Session, Posters \& Demonstrations

[17] Rokita JL, Rathi KS, Cardenas MF, et al. Genomic Profiling of Childhood Tumor Patient-Derived Xenograft Models to Enable Rational Clinical Trial Design. Cell Rep. 2019;29(6):1675-1689.e9. doi:10.1016/j.celrep.2019.09.071

[18] Upton K, Modi A, Patel K, et al. Epigenomic profiling of neuroblastoma cell lines. Sci Data. 2020;7(1):116. Published 2020 Apr 14. doi:10.1038/s41597-020-0458-y

[19] Kendsersky NM, Lindsay J, Kolb EA, et al. The B7-H3-Targeting Antibody-Drug Conjugate m276-SL-PBD Is Potently Effective Against Pediatric Cancer Preclinical Solid Tumor Models. Clin Cancer Res. 2021;27(10):29382946. doi:10.1158/1078-0432.CCR-20-4221

[20] Rushabh Patel and Rahul Raghvendra Joshi, Envision of I-RS (I-Railway System) - based on Cloud Computing, International Journal of Science Engineering and Technology Research (IJSETR), vol. 4, no. 1, January 2015.

[21] Yang X, He H. An advanced compiler designed for a VLIW DSP for sensors-based systems. Sensors (Basel). 2012;12(4):4466-4478. doi:10.3390/s120404466

[22] Rushabh Patel and Rahul Raghvendra Joshi, Introducing a Hi-Tech - Cloud based Public Transport System, International Journal of Computational Intelligence Research ISSN 0973-1873 Volume 11, Number 1 (2015), pp. 1926

[23] Raman, Swetha and Buongervino, Samantha N. and Lane, Maria and Zhelev, Doncho V. and Zhu, Zhongyu and Cui, Hong and Martinez, Benjamin and Martinez, Daniel and Wang, Yanping and Upton, Kristen A. and Patel, Khushbu and Rathi, Komal S. and Navia, Carmen T. and Harmon, Daniel B. and Pawel, Bruce and Dimitrov, Dimiter and Maris, John M. and Julien, Jean-Philippe and Bosse, Kristopher, The D3-GPC2-PBD ADC is Potently Efficacious Against Neuroblastoma and SCLC Via Engagement of a Conformational GPC2 Epitope (September 2020). Available at SSRN: https://ssrn.com/abstract=3673604 or http://dx.doi.org/10.2139/ssrn.3673604 